\title{
High seroprevalence of syphilis infection among pregnant women in Yiregalem hospital southern Ethiopia
}

\author{
Anteneh Amsalu ${ }^{1 *}$, Getachew Ferede ${ }^{1}$ and Demissie Assegu ${ }^{2}$
}

\begin{abstract}
Background: Despite availability of effective treatment and the implementation of focused antenatal care (ANC), still the prevalence of syphilis persists in Ethiopia. Yet, data is not found in southern Ethiopia. Therefore, this study was conducted to determine the seroprevalence and associated factors of syphilis among pregnant women in Yiregalem Hospital, Southern Ethiopia.
\end{abstract}

Methods: A cross-sectional study was conducted among pregnant women from October 2015 to August 2016. Data on socio-demography and obstetric conditions of pregnant women were collected using a structured questionnaire. Serum samples were screened for syphilis using rapid plasma regain (RPR) test and those found seropositive were further confirmed by Treponema pallidum haemagglutination assay (TPHA) test following the manufacturer's instruction. HIV results were reviewed from records. The data were analyzed using SPSS version 20 software.

Results: Of 494 pregnant women, 204(41.3\%) were first ANC visit attenders. Of these, 123(60.3\%) were after the 12th gestational week. Sero-prevalence of syphilis and HIV was found to be 5.1\% (25/494; 95 Cl, 3.2-7.1\%) and 10.3\% (51/494; $95 \mathrm{Cl}, 7.7-13.2 \%)$, respectively. The overall prevalence of syphilis and HIV co-infection was 2.2\% (11/494). Women with HIV infection were significantly more likely to be syphilis-seropositive ( $A O R=10.3,95 \% \mathrm{Cl}, 4.213-25.185)$ than HIV negatives.

Conclusions: High seroprevalence of syphilis particularly among HIV positive women and late first ANC visit attenders in the study area calls for further ramping up of current intervention measures. Like integration of syphilis testing and treatment to the already established HIV prevention program and creating awareness about early ANC visit and follow-up.

Keywords: Syphilis, Pregnant women, Ethiopia

\section{Background}

Despite, widely available and affordable screening methods and treatment, syphilis remains a public health problem in pregnant women worldwide particularly in sub-Saharan Africa (SSA) [1, 2]. An estimated 2.7\% (0.1-10.3\%) of pregnant women in SSA are infected with syphilis, representing more than 900,000 pregnancies at risk each year [1]. In Ethiopia, according to ANC-based sentinel surveillances, syphilis prevalence increased from $1 \%$, in 2012 to $1.2 \%$, in 2014 [3] and as high as 2.9 to $3.7 \%$ in recent studies among ANC attendees in Gondar [4-6] and 7.3 to $9.8 \%$ among

\footnotetext{
* Correspondence: ant.amsalu@gmail.com

${ }^{1}$ Department of Medical Microbiology, University of Gondar, P.O.Box 196,

Gondar, Ethiopia

Full list of author information is available at the end of the article
}

HIV patients in Hawassa [7] and Addis Ababa [8], respectively.

Syphilis is a chronic infectious disease caused by the Spirochaete, Treponema pallidum. It causes ulcerative genital lesion that enhances the acquisition and sexual transmission of HIV infection [2]. It has been shown that maternal syphilis can increase HIV transmission during sexual intercourse, in utero or at delivery by twoto- fivefold $[9,10]$. Patients with concurrent HIV infection are thought to be at increased risk of neurological complications and treatment failure [11]. Kuznik et al. reported an estimate of 205,901 adverse pregnancy outcomes, such as spontaneous abortion, stillbirth, low birth weight, neonatal death and congenital syphilis, due to untreated maternal syphilis [12]. Ethiopia is among the three SSA countries with the highest numbers of 
adverse pregnancy outcomes [12]. Due to these adverse outcomes.

The World Health Organization (WHO) recommends that the first antenatal visit should be initiated at $\leq 12$ weeks and at least four ANC visits are required at specified intervals for healthy pregnant women with no underlying medical problems, in focused antenatal care [13]. Importantly, control of vertical transmission of syphilis and associated birth complications is possible through timely screening of all pregnant mothers at the first prenatal visit and those who are at high risk for syphilis, are previously untested, or live in areas of high syphilis prevalence should be screened again early in the third trimester and at delivery and treated if positive $[14,15]$. However, in practice, there is considerable screening and therapeutic heterogeneity. In Ethiopia, pregnant women are tested only once for syphilis during ANC visits using rapid plasma reagin (RPR) test and those women reactive for RPR were treated immediately with one or more dose of intramuscular benzathine penicillin G 2.4 million units [16]. However, the burden of this disease in pregnancy persists $[5,6]$ and the burden may vary depending on the variation in the timing and frequency of ANC visit, diagnosis and treatment, socio-demographic variables, cultural practice and socio-economic factors [17]. Such data is scarce in Ethiopia [5, 6], particularly in the study area. Therefore, this study was aimed to provide information for health policy makers to plan effective strategies to control syphilis transmission during pregnancy.

\section{Methods}

\section{Study design, area and period}

A cross sectional study was conducted among pregnant women attending in Yirgalem hospital ANC clinic from October 2015 to August 2016. The hospital was located $70 \mathrm{~km}$ from Hawassa, the capital city of Southern Nations and Nationalities People's Region (SNNPR) and $273 \mathrm{~km}$ from Addis Ababa, the capital city of Ethiopia. It is the largest hospital in Southern Ethiopia and provides medical education and training in addition to medical care. The hospital ANC clinic gives prenatal, delivery and postnatal care services for pregnant women and has 69 bed rooms for pregnant women. HIV and syphilis screening (RPR test only) for pregnant womenis offered at the time of their first ANC visit in the hospital.

\section{Population}

The study population consisted of pregnant women attending ANC clinic in Yirgalem hospital during the study period. Women less than 18 years of age, on treatment for syphilis and labor or delivery during recruitment were excluded. Sample size was estimated to be 342 using single population proportion formula, assuming $3.7 \%$ syphilis prevalence in pregnant women in Gondar, Ethiopia [5]; 2\% precision and 95\% level of confidence. But, in attempting to enhance the statistical power of detecting the rate difference by exposure status, we investigated a total of 500 pregnant women, prospectively. Study populations were selected using systematic random sampling techniques; the first one pregnant woman was selected randomly out of 5 women visiting the hospital and then every 5th women was enrolled in the study until the total sample was achieved. Code number was written with special mark on their follow-up chart to avoid duplication during the study period.

\section{Data collection \\ Socio-demographic data}

After providing written informed consent, participants answered a questionnaire that included questions about their socio-demography and obstetric history. In addition, HIV result and antiretroviral therapy (ART) status were also obtained from their follow-up chart by two midwife nurses.

\section{Laboratory testing}

Five milliliter of blood samples were collected and screened for syphilis using rapid plasma reagin (RPR) test (Human, Germany). Sera tested positive by RPR tests were confirmed by modified Treponema pallidum haemagglutination assay (TPHA) (Syphicheck-WB, Qualpro Diagnostics, India). Laboratory tests were carried out according to the instructions of the manufacturers and all tests were run against the positive and negative controls. Only those samples positive by both RPR and TPHA were considered as probable active syphilis (PAS) infection [18].

\section{Data analysis}

Data were coded, entered and analyzed using SPSS version 20(IBM Corp., Armonk, NY, USA). We described data using either proportion or mean with standard deviation (SD). The association between syphilis infection and socio-demographic factors were assessed using crude odds ratio (COR) from a binary logistic regression analysis. Adjusted odds ratio (AOR) was also computed using multivariable logistic regression analysis, taking all factors yielding a $p$-value $\leq 0.2$ in bivariate analysis. A $p$-value of $<0.05$ was accepted as significant.

\section{Ethical consideration}

This study was reviewed and approved by the Institutional Review Board (IRB) of Hawassa University College of Medicine and Health Science. A letter of support was obtained from the SNNPR health bureau and Yirgalem hospital administration. Informed written consent was obtained from the study participants and any information 
obtained during the study was kept with utmost confidentiality, and those found infected with syphilis were treated.

\section{Results}

Socio-demographic characteristics of study population Out of the 500 pregnant women, 494 (98.8\%) were enrolled in the study, while the rest $6(1.2 \%)$ were excluded from the analysis due to incomplete questionnaire. The mean age of pregnant women was 26.5 years (standard deviation [SD], 4.6; range, 18-42 years). Majority $447(90.5 \%)$ of study participants were married and had at least primary education $435(88.1 \%)$. Greater than two thirds $(67.4 \%)$ of the pregnant women were urban residents and 246(49.8\%) were housewives. Their previous obstetric history revealed a mean of 2.4 gravida (ranges from 1 to 9) and 334(67.6\%) of women were multigravida, $239(71.6 \%)$ had history of institutional delivery (Table 1).

\section{Gestational age with frequency of antenatal care visit} Out of 494 women who attended ANC during the study period, 204(41.3\%) were first visit attendees. Of these, only $81(39.7 \%)$ were in the first trimester while

Table 1 Socio-demography and some obstetric conditions of pregnant women at Yirgalem hospital, Southern Ethiopia, 2016

\begin{tabular}{|c|c|c|c|}
\hline Characteristics & & $\begin{array}{l}\text { Number } \\
(n=494)\end{array}$ & $\begin{array}{l}\text { Percent } \\
(\%)\end{array}$ \\
\hline \multirow[t]{3}{*}{ Age(years) } & $<25$ & 161 & 32.6 \\
\hline & $25-29$ & 214 & 43.3 \\
\hline & $\geq 30$ & 119 & 24.1 \\
\hline \multirow[t]{2}{*}{ Residence } & Urban & 333 & 67.4 \\
\hline & Rural & 161 & 32.6 \\
\hline \multirow[t]{4}{*}{ Education } & Illiterate & 59 & 11.9 \\
\hline & Primary & 168 & 34.0 \\
\hline & Secondary & 166 & 33.6 \\
\hline & Tertiary & 101 & 20.4 \\
\hline \multirow[t]{5}{*}{ Occupation } & House wife & 246 & 49.8 \\
\hline & Health worker & 19 & 3.8 \\
\hline & Employed & 133 & 26.9 \\
\hline & Merchant & 52 & 10.5 \\
\hline & Student & 44 & 8.9 \\
\hline \multirow[t]{2}{*}{ Marital status } & Married & 447 & 90.5 \\
\hline & $\begin{array}{l}\text { Single/divorced/ } \\
\text { widowed }\end{array}$ & 47 & 9.5 \\
\hline \multirow[t]{2}{*}{ Gravidity } & Primigravida & 160 & 32.4 \\
\hline & Multigravida( $\geq 2$ ) & 334 & 67.6 \\
\hline $\begin{array}{l}\text { Place of previous } \\
\text { birth }\end{array}$ & Home & 95 & 28.4 \\
\hline $\begin{array}{l}\text { (excludes } \\
\text { primigravida) }\end{array}$ & Health institution & 239 & 71.6 \\
\hline
\end{tabular}

123(60.3\%) were late (in the second and third trimester) attendees. Considering, gestational age the majority (43.2\%) of women were in the third trimester. But, only 73(34.1\%) out of 214 ANC attenders in the third trimester had attended at least four ANC visits (Table 2).

\section{Seroprevalence of syphilis}

Of 494 pregnant women, the prevalence of confirmed syphilis seropositive was $5.1 \%$ (25/494; 95 CI, 3.2-7.1\%). Twenty-five among 30 participants $(6.1 \%)$ with a positive RPR were also positive for TPHA. The overall HIV prevalence was found to be $10.3 \%(51 / 494 ; 95 \mathrm{CI}, 7.7$ $-13.2 \%)$. Among them, sixteen (31.4\%) women knew their HIV status for the first time while, $68.6 \%(35 / 51)$ they knew their HIV status before the study period and had started ART. The overall prevalence of syphilis-HIV co-infection was $2.2 \%(11 / 494)$ (Table 3).

\section{Associated factors of syphilis infection}

Syphilis prevalence increased with increasing age, with the highest rate in the age $\geq 30$ years (5.9\%). Similarly, seroprevalence of syphilis increased with gestational period, peak in third trimester $(6.1 \%)$ and also in women who were occupationally health worker (10.5\%), followed by housewives $(6.1 \%)$, rural resident( $6.3 \%)$, not cohabiting with their partner $(8.5 \%)$, multigravidae (5.4\%), had more than one ANC visit (5.9\%), multiple sexual partner(8.9\%), previous history of home delivery $(6.3 \%)$ and HIV positive (21.6\%)(Table 4).

In multivariate logistic regression analysis, women with HIV infection were significantly more likely to be syphilis-seropositive (AOR =10.3, 95\%CI, 4.213-25.185, $p<0.001)$ as compared to HIV negatives. However, age, residence, occupation, marital status, total number of ANC visit, gravidity, multiple sexual factors and previous place of birth were not statistical significant (Table 4).

Table 2 The total number of ANC visits and gestational age at the final screening of pregnant women inYirgalem hospital, Southern Ethiopia, 2016

\begin{tabular}{lllll}
\hline $\begin{array}{l}\text { Total number } \\
\text { of ANC visits }\end{array}$ & \multicolumn{2}{l}{ Gestational age at final screening } & \\
\cline { 2 - 4 } & $\begin{array}{l}1^{\text {st }} \text { trimester } \\
\mathrm{n}(\%)\end{array}$ & $\begin{array}{l}2^{\text {nd }} \text { trimester } \\
\mathrm{n}(\%)\end{array}$ & $\begin{array}{l}3^{\text {rd }} \text { trimester } \\
\mathrm{n}(\%)\end{array}$ & $\begin{array}{l}\text { Total } \\
\mathrm{n}(\%)\end{array}$ \\
\hline 1 & $81(39.7)$ & $79(38.7)$ & $44(21.6)$ & $204(41.3)$ \\
2 & $14(12.6)$ & $64(57.7)$ & $33(29.7)$ & $111(22.5)$ \\
3 & $5(5.1)$ & $30(30.3)$ & $64(64.6)$ & $99(20.0)$ \\
$\geq 4$ & 0 & $7(8.8)$ & $73(91.2)$ & $80(16.2)$ \\
Total & $100(20.2)$ & $180(36.5)$ & $214(43.3)$ & $494(100)$ \\
\hline
\end{tabular}

ANC antenatal care 
Table 3 Syphilis serological tests in pregnant women by HIV status at Yirgalem hospital, Southern Ethiopia, 2016

\begin{tabular}{llll}
\hline Syphilis test & $\begin{array}{l}\text { Total } \\
\text { tested }\end{array}$ & $\begin{array}{l}\mathrm{n}(\%) \text { syphilis } \\
\text { positive }\end{array}$ & $\begin{array}{l}\mathrm{n}(\%) \text { HIV positive } \\
(\mathrm{N}=51 / 494)\end{array}$ \\
\hline RPR & 494 & $30(6.1)$ & $14(2.8)$ \\
TPHA & 30 & $25(83.3)$ & $11(36.7)$ \\
Syphilis seropositivity & 494 & $25(5.1)$ & $11(2.2)$ \\
\hline
\end{tabular}

HIV human immunodeficiency virus, $n$ total number of syphilis positive, $N$ total number of HIV positive, RPR rapid plasma regain, TPHA Treponema pallidum haemagglutination assay

\section{Discussion}

The seroprevalence of syphilis among pregnant women in this study was $5.1 \%$, which is comparable to the studies conducted in Gondar, Ethiopia (3.7\%) [5] and Uganda (5.1\%) [19]. In contrast, it is higher than that found in the national sentinel surveillance studies in 2012(1\%) and $2014(1.2 \%)$ [3] and previous studies in Gondar, Ethiopia (1-2.9\%) [4, 6, 20, 21], Tanzania (1.6-2.5\%) [22, 23] and Madagascar (3\%) [24]. Moreover, higher seroprevalence of syphilis was also reported among ANC attendees in Tanzania(7.2\%) [25], and Zambia(8.2\%) [26] and in HIV patients in different parts of Ethiopia(7.3-9.8\%) [7, 8]. The observed differences might be due to differences in geographic sites and time-period, the data source (primary or secondary data), socio-cultural and economic factors, and differential access to syphilis diagnosis and treatment.

In this study, syphilis seroprevalence increased slightly with increasing age which is in agreement with the studies conducted elsewhere $[5,7,24,27]$. This is perhaps due to the risk of exposure to syphilis increased with time. The preponderance of syphilis among women not cohabiting with their partner and urban residence in our study was concordant with findings reported in Gondar [4]. In contrast, high burden of syphilis in the rural area has been reported in the studies from Gondar, Ethiopia [6] and Tanzania [22]. The high burden of syphilis in the urban residents may be partially explained by $68.6 \%$ of HIV positive women in our study were urban dwellers as those infections share the same risk factor and one facilitate the presence of the other; as well as commercial sexual practice is relatively more permissible in urban than rural population [28].

In this study, high prevalence of HIV infection (10.3\%) among pregnant women attending the ANC was consistent with the previous studies in Gondar (9.6-11.9\%) [5, 6, 20, 21]. Women with HIV infection were significantly more likely to be syphilis-seropositive than HIV negatives concordant with the national sentential surveillance [3] and other studies [29, 30]. The overall syphilis/HIV co-infection rate in our study population was $2.2 \%$. This co-infection rate is about two to four times higher than the rate recently reported among pregnant women in Gondar [5, 6]. This calls for strengthening the integration of syphilis screening in the established antenatal HIV prevention programs [31] and implementation of rapid dual HIV/syphilis screening test in ANC clinics in Ethiopia to improve syphilis and HIV screening coverage, early detection, and maternal treatment to prevent infant infection $[9,30]$.

According to $\mathrm{WHO}$, delayed antenatal care (after the 12th gestational week) is one of the barriers for the control of adverse pregnancy outcomes [14]. It is associated with fewer medical consultations and fewer routine examinations particularly in resource limited countries like Ethiopia where non-specific (RPR) test is widely used. The test may not indicate syphilis diagnosis in a single visit and perhaps missed the positive cases particularly in the presence of concurrent HIV infection. In the present study, delayed antenatal care was observed in $60.3 \%$ of pregnant women in the first ANC visit. It is an indicative that many pregnant women may have been diagnosed and treated after the recommended gestational age and are more likely to attain poor outcomes of pregnancy [14]. Additionally, high seroprevalence of syphilis in those women who visited ANC services more than once and women at third trimester suggests that those women visited the clinic more than once may either not properly screened or treated in their first ANC visit. Taken together, this observation highlights the need to strengthen the existing ANC services to reduce mother to child transmission of syphilis [11].

Nevertheless, sample size limited our ability to show significance for many demographic variables. We did not evaluate birth outcomes of mothers treated for syphilis due to the cross sectional nature of the study. We did not also report on syphilis screening and treatment coverage within pregnant women seen at this facility overall. Regardless of the depicted limitations, this study ultimately adds supportive information on increased prevalence of syphilis in the study area.

\section{Conclusions}

The seroprevalence of syphilis among pregnant women in this hospital in Southern Ethiopia was considerably higher than in previous studies in Ethiopia. Women with HIV infection were significantly more likely to be syphilis-seropositive. Syphilis/HIV co-infection coupled with other gaps in the health care, such as lack of specific diagnostic tests and delayed ANC visits in Ethiopia; contribute to the persistence of syphilis as a major public health problem. Therefore, it is necessary to design strategies for health professionals in order to assure the minimum number of visits required by the Ethiopian Ministry of Health. In addition, timely access to dual 
Table 4 Associated factors of syphilis infection among pregnant women at Yirgalem hospital, Southern Ethiopia, 2016

\begin{tabular}{|c|c|c|c|c|c|c|}
\hline \multirow[t]{2}{*}{ Variables } & & \multicolumn{2}{|l|}{ Syphilis } & \multirow[t]{2}{*}{ COR $(95 \% \mathrm{Cl})$} & \multirow[t]{2}{*}{ AOR $(95 \% C l)$} & \multirow[t]{2}{*}{$P$-value } \\
\hline & & $\mathrm{NR}(n=469)$ & $\mathrm{R}(n=25)$ & & & \\
\hline \multirow[t]{3}{*}{ Age(years) } & $<25$ & $155(96.3)$ & $6(3.7)$ & 1 & & \\
\hline & $25-29$ & $202(94.4)$ & $12(5.6)$ & $1.5(0.563-4.180)$ & & \\
\hline & $\geq 30$ & $112(94.1)$ & $7(5.9)$ & $1.6(0.528-4.935)$ & & \\
\hline \multirow[t]{2}{*}{ Residence } & Urban & $312(93.7)$ & $21(6.3)$ & $2.6(0.892-7.829)^{* *}$ & $2.6(0.845-7.982)$ & 0.096 \\
\hline & Rural & $157(97.5)$ & $4(2.5)$ & 1 & & \\
\hline \multirow[t]{4}{*}{ Education } & Illiterate & $57(96.6)$ & $2(3.4)$ & 1 & & \\
\hline & Elementary & $160(95.2)$ & $8(4.8)$ & $1.4(0.294-6.909)$ & & \\
\hline & Secondary & $156(94.0)$ & $10(6.0)$ & $1.8(0.388-8.592)$ & & \\
\hline & Tertiary & $96(95.0)$ & $5(5.0)$ & $1.5(0.279-7.903)$ & & \\
\hline \multirow[t]{5}{*}{ Occupational } & House wife & $231(93.9)$ & $15(6.1)$ & $2.8(0.359-21.695)$ & & \\
\hline & Health worker & $17(89.5)$ & $2(10.5)$ & $5.0(0.430-59.525)$ & & \\
\hline & Employed & $128(96.2)$ & $5(3.8)$ & $1.7(0.191-14.780)$ & & \\
\hline & Merchant & $50(96.2)$ & $2(3.8)$ & $1.7(0.151-19.632)$ & & \\
\hline & Student & $43(97.7)$ & $1(2.3)$ & 1 & & \\
\hline \multirow[t]{2}{*}{ Marital status } & Married & $426(95.3)$ & $21(4.7)$ & 1 & & \\
\hline & Not cohabiting* & $43(91.5)$ & $4(8.5)$ & $1.9(0.619-5.751)$ & & \\
\hline \multirow[t]{3}{*}{ Gestational age at screening } & First trimester & $98(98.0)$ & $2(2.0)$ & 1 & & \\
\hline & Second trimester & $170(94.4)$ & $10(5.6)$ & $2.9(0.619-13.423)^{* *}$ & $3.8(0.778-18.847)$ & 0.099 \\
\hline & Third trimester & $201(93.9)$ & $13(6.1)$ & $3.2(0.701-14.319)^{* *}$ & $4.6(0.959-22.340)$ & 0.056 \\
\hline \multirow[t]{2}{*}{ Gravidity } & Primigravidae & $153(95.6)$ & $7(4.4)$ & 1 & & \\
\hline & Multigravidae( $\geq 2)$ & $316(94.6)$ & $18(5.4)$ & $1.2(0.509-3.044)$ & & \\
\hline Frequency of & 1 & $196(96.1)$ & $8(3.9)$ & 1 & & \\
\hline ANC visit & $\geq 2$ & $273(94.1)$ & $17(5.9)$ & $1.5(0.646-3.606)$ & & \\
\hline Multiple sexual & Yes & $41(91.1)$ & $4(8.9)$ & $2.0(0.651-6.701)$ & & \\
\hline Partner & No & $428(95.3)$ & $21(4.7)$ & 1 & & \\
\hline Place of & Home & $89(93.7)$ & $6(6.3)$ & $1.3(0.464-3.502)$ & & \\
\hline previous birth & Health institution & $227(95.0)$ & $12(5.0)$ & 1 & & \\
\hline \multirow[t]{2}{*}{ HIV } & Non-reactive & $429(96.8)$ & $14(3.2)$ & 1 & & \\
\hline & Reactive & $40(79.4)$ & $11(21.6)$ & $8.4(3.859-19.786)^{* *}$ & $10.3(4.213-25.185)$ & 0.001 \\
\hline
\end{tabular}

*Not cohabiting, ${ }^{*}$ single/divorced /widowed; $p$-value $<0.2 ; R$ reactive, NR nonreactive, COR crude odds ratio, AOR adjusted odds ratio

detection of HIV and syphilis and treatment for those tested positive for syphilis should be of extreme concern and priority in the control of syphilis. Furthermore, associated factors for syphilis transmission need to be further studied in large sample size in different health centre in the region and in the country at large, which will be helpful to tailor the current health education programs at antenatal care clinics to raise the awareness of mothers.

\section{Abbreviations}

ANC: Antenatal care; ART: Antiretroviral therapy; HIV: Human

immunodeficiency virus; RPR: Rapid plasma regain; SNNPR: Southern Nations and Nationalities People's Region; SSA: Sub-Saharan Africa; TPHA: Treponema pallidum haemagglutination assay; WHO: World Health Organization

\section{Acknowledgements}

The authors would like to thank the study participants who took part in this study and the data collectors. We are also most grateful to Hawassa University for financial support and Yirgalem hospital administration for allowing us to conduct this research.

\section{Funding}

The study was supported with a small grant obtained from Hawassa University.

\section{Availability of data and materials}

The datasets used and/or analysed during the current study are available from the corresponding author on reasonable request.

\section{Authors' contributions}

AA conceived the idea and designed the study; DA carried out the laboratory work; AA, GF and DA performed the statistical analyses and interpretation and contributed to the write-up. All authors read and approved the final version of the manuscript. 


\section{Ethics approval and consent to participate}

The study was approved by the Institutional Review Board of the College of Medicine and Health Sciences, Hawassa University and informed written consent was obtained from the study participants.

\section{Consent for publication}

Not applicable.

\section{Competing interests}

The authors declare that they have no computing interests.

\section{Publisher's Note}

Springer Nature remains neutral with regard to jurisdictional claims in published maps and institutional affiliations.

\section{Author details}

'Department of Medical Microbiology, University of Gondar, P.O.Box 196, Gondar, Ethiopia. ${ }^{2}$ Department of Medical Laboratory Sciences, Hawassa University, Hawassa, Ethiopia.

Received: 30 August 2017 Accepted: 16 February 2018

Published online: 06 March 2018

\section{References}

1. World Health Organization. Baseline report on global sexually transmitted infection surveillance 2013. Geneva (Switzerland): World Health Organization; 2014.

2. Karp G, Schlaeffer F, Jotkowitz A, Riesenberg K. Syphilis and HIV co-infection. Eur J intern med. 2009;20(1):9-13.

3. The Ethiopian Public Health Institute: Report on the 2014 round antenatal care based sentinel HIV surveillance in Ethiopia. 2015

4. Assefa A. A three year retrospective study on Seroprevalence of syphilis among pregnant women at Gondar University teaching hospital, Ethiopia. Afr Health Sci. 2014;14(1):119-24. https://doi.org/10.4314/ahs.v14i1.18

5. Melku M, Kebede A, Addis Z. Magnitude of HIV and syphilis seroprevalence among pregnant women in gondar, northwest ethiopia: a cross-sectional study. HIV/AIDS. 2015;7:175.

6. Endris M, Deressa T, Belyhun Y, Moges F. Seroprevalence of syphilis and human immunodeficiency virus infections among pregnant women who attend the University of Gondar teaching hospital, Northwest Ethiopia: a cross sectional study. BMC Infect Dis. 2015;15(1):111.

7. Shimelis T, Lemma K, Ambachew H, Tadesse E. Syphilis among people with HIV infection in southern Ethiopia: sero-prevalence and risk factors. BMC Infect Dis. 2015;15(1):189.

8. Eticha BT, Sisay Z, Alemayehu A, Shimelis T. Seroprevalence of syphilis among HIV-infected individuals in Addis Ababa, Ethiopia: a hospital-based cross-sectional study. BMJ Open. 2013;3(4):e002566.

9. Taylor MM, Peeling RW, Toskin I, Ghinidelli M. Role of dual HIV/syphilis test kits in expanding syphilis screening. Sex Transm Infect. 2017;0:1-2. https:// doi.org/10.1136/sextrans-2017-053301.

10. Mwapasa V, Rogerson SJ, Kwiek JJ, Wilson PE, Milner D, Molyneux ME, Kamwendo DD, Tadesse E, Chaluluka E, Meshnick SR. Maternal syphilis infection is associated with increased risk of mother-to-child transmission of HIV in Malawi. AIDS. 2006;20(14):1869-77.

11. WHO: Guidline on syphilis screening and treatment for pregnant women. 2017: http://apps.who.int/iris/bitstream/10665/259003/1/9789241550093-eng.pdf.

12. Kuznik A, Habib AG, Manabe YC, Lamorde M. Estimating the public health burden associated with adverse pregnancy outcomes resulting from syphilis infection across 43 countries in sub-Saharan Africa. Sex Transm Dis. 2015; 42(7):369.

13. Lincetto O, Mothebesoane-Anoh S, Gomez P, Munjanja S. Chapter 2: Antenatal care. In: Lawn J, Kerber K, editors.Opportunities for Africa's Newborns: practical data, policy and programmatic support for newborn care in Africa. Geneva: World Health Organization; 2006. pp. 51-62. http:// www.who.int/pmnch/media/publications/aonsectionlll_2.pdf. Accessed 7 Aug 2017.

14. Hawkes SJ, Gomez GB, Broutet N. Early antenatal care: does it make a difference to outcomes of pregnancy associated with syphilis? A systematic review and meta-analysis. PLoS One. 2013;8(2):e56713. https://doi.org/10. 1371/journal.pone.0056713.
15. Centers for Disease Control and Prevention: Sexually transmitted diseases treatment guidelines 2015:https:/www.cdc.gov/std/tg2015/tg-2015-print.pdf.

16. Federal Democratic Republic of Ethiopia Ministry of Health: National guidelines for the management of sexually transmitted infections using syndromic approach. 2015.

17. Mani SB, Candidate M, Pegany R, Sheng D: Maternal Syphilis: Variations in Prenatal Screening, Treatment, and Diagnosis of Congenital Syphilis. Col Med Rev. 2017;1(2):20-29. https://doi.org/10.7916/D8475NSR.

18. Ham DC, Lin C, Newman L, Wijesooriya NS, Kamb M. Improving global estimates of syphilis in pregnancy by diagnostic test type: a systematic review and meta-analysis. Int J Gynecol Obstet. 2015;130:S10-4.

19. Manabe YC, Namale G, Nalintya E, Sempa J, Ratanshi RP, Pakker N, Katabira E. Integration of antenatal syphilis screening in an urban HIV clinic: a feasibility study. BMC Infect Dis. 2015;15(1):15.

20. Andargie G, Kassu A, Moges F, Kebede Y, Gedefaw M, Wale F, Alem A Andualem B, Adungna S. Brief communication: low prevalence of HIV infection, and knowledge, attitude and practice on HIV/AIDS among high school students in Gondar, Northwest Ethiopia. Ethiop J Health Dev. 2007; 21(2):179-82.

21. Tiruneh M. Seroprevalence of multiple sexually transmitted infections among antenatal clinic attendees in Gondar health center, northwest Ethiopia. Ethiop Med J. 2008:46(4):359-66.

22. Manyahi J, Jullu BS, Abuya MI, Juma J, Ndayongeje J, Kilama B, Sambu V, Nondi J, Rabiel B, Somi G. Prevalence of HIV and syphilis infections among pregnant women attending antenatal clinics in Tanzania, 2011. BMC Public Health. 2015;15(1):501.

23. Yahya-Malima Kl, Evjen-Olsen B, Matee MI, Fylkesnes K, Haarr L. HIV-1, HSV-2 and syphilis among pregnant women in a rural area of Tanzania: prevalence and risk factors. BMC Infect Dis. 2008;8(1):75

24. Frickmann H, Schwarz NG, Girmann M, Hagen RM, Poppert S, Crusius S, Podbielski A, Heriniaina JN, Razafindrabe T, Rakotondrainiarivelo JP. Serological survey of HIV and syphilis in pregnant women in Madagascar. Tropical Med Int Health. 2013:18(1):35-9.

25. Ministry of Health Tanzania/National AIDS Control Program (NACP): Surveillance of HIV and Syphilis Infection Among Antenatal Clinic Attendees 2003/2004. 2005 Apr. report.

26. Central Statistical Office/Zambia, Central Board of Health/Zambia, ORC Macro. Zambia Demographic and Health Survey 2001-2002. Calverton: Central Statistical Office/Zambia, Central Board of Health/Zambia, and ORC Macro; 2003.

27. Yang L-G, Tucker JD, Liu F-Y, Ren X-Q, Hong X, Wang C, McLaughlin MM, Bien $\mathrm{CH}$, Chen X-S, Yang B. Syphilis screening among 27,150 pregnant women in south Chinese rural areas using point-of-care tests. PLoS One. 2013:8(8):e72149.

28. WHO: Information note on the use of dual HIV/syphilis rapid diagnostic tests (RDT). 2017:http://apps.who.int/iris/bitstream/10665/252849/1/WHORHR-17.01-eng.pdf.

29. Potter D, Goldenberg RL, Read JS, Wang J, Hoffman IF, Saathoff E, Kafulafula G, Aboud S, Martinson FE, Dahab M. Correlates of syphilis seroreactivity among pregnant women: the HIVNET 024 trial in Malawi, Tanzania, and Zambia. Sex Transm Infect. 2006;33(10):604.

30. Gliddon H, Peeling R, Kamb ML, Toskin I, Taylor MM. A systematic review and meta-analysis of studies evaluating the performance and operational characteristics of dual point-of-care tests for HIV and syphilis. Sex Transm Infect. 2017;0:1-13. https://doi.org/10.1136/sextrans-2016-053069.

31. Taylor MM, Kamb ML, Wu D, Hawkes S. Syphilis screening and treatment: integration with HIV services. Bull World Health Organ. 2017;95(9):610-610A. https://doi.org/10.2471/BLT.17.200923. 